\title{
Simulations Study of Muon Response in the Peripheral Regions of the Iron Calorimeter Detector at the India-based Neutrino Observatory
}

\author{
R. Kanishka*, Meghna K. K. ${ }^{\dagger}$, \\ Vipin Bhatnagar*, D. Indumathi ${ }^{\dagger}$, Nita Sinha ${ }^{\dagger}$ \\ * Physics Department, Panjab University, Sector 14, Chandigarh 160 014, India \\ ${ }^{\dagger}$ The Institute of Mathematical Sciences, CIT Campus, Chennai 600 113, India
}

August 7, 2018

\begin{abstract}
The magnetized Iron CALorimeter detector (ICAL) which is proposed to be built in the India-based Neutrino Observatory (INO) laboratory, aims to study atmospheric neutrino oscillations primarily through charged current interactions of muon neutrinos and anti-neutrinos with the detector. The response of muons and charge identification efficiency, angle and energy resolution as a function of muon momentum and direction are studied from GEANT4-based simulations in the peripheral regions of the detector. This completes the characterisation of ICAL with respect to muons over the entire detector and has implications for the sensitivity of ICAL to the oscillation parameters and mass hierarchy compared to the studies where only the resolutions and efficiencies of the central region of ICAL were assumed for the entire detector.

Selection criteria for track reconstruction in the peripheral region of the detector were determined from the detector response. On applying these, for the 1-20 GeV energy region of interest for mass hierarchy studies, an average angle-dependent momentum resolution of $15-24 \%$, reconstruction efficiency of about $60-70 \%$ and a correct charge identification of about $97 \%$ of the reconstructed muons were obtained. In addition, muon response at higher energies upto $50 \mathrm{GeV}$ was studied as relevant for understanding the response to so-called rock muons and cosmic ray muons. An angular resolution of better than a degree for muon energies greater than $4 \mathrm{GeV}$ was obtained in the peripheral regions, which is the same as that in the central region.
\end{abstract}

*email: kanishka@puhep.res.in 


\section{Introduction}

The India-based Neutrino Observatory (INO) [1] is the proposed underground facility that will house a magnetized Iron CALorimeter detector (ICAL) designed to study neutrino oscillations with atmospheric muon neutrinos. In particular, ICAL will focus on measuring precisely the neutrino oscillation parameters including the sign of the 2-3 mass-squared difference $\Delta m_{32}^{2}$ $\left(=m_{3}^{2}-m_{2}^{2}\right)$ and hence help to determine the neutrino mass hierarchy through matter effects. Oscillation signatures for neutrinos and anti-neutrinos are different in the presence of matter effects which become important in the few GeV energy region. These parameters are sensitive to the momentum $P$ and the zenith angle $\cos \theta$ (through path length travelled) of neutrinos. Reconstruction of these parameters further depends on the energy and direction of muons and hadrons 2 2] produced in charge-current interactions of the neutrinos in the detector; hence studies of muon energy and direction resolutions are crucial.

Since ICAL is designed to be mostly sensitive to muons, the main physics issues that ICAL will probe will be through studies of charged current (CC) muon neutrino (anti-neutrino) interactions with muons (anti-muons) produced in the final state. Two types of interactions are relevant: in the first, the neutrino enters the detector, interacting with (dominantly) nucleons in the iron nucleus. These events are identified by vertices which are inside the detector (tracks begin inside the detector). In the second type of interaction, the neutrino interacts with rock around the detector and the produced muons lose energy while propagating through the rock (these are the so-called rock muons or upward-going muons). These events have vertices outside the detector with their tracks starting at an edge of the detector. The first type of interaction dominantly gives low energy (few $\mathrm{GeV}$ ) muons due to the rapidly falling atmospheric neutrino flux. While in the case of rock muons, most of the lower energy muons are stopped in the rock itself, so that the fraction of higher energy $(>10 \mathrm{GeV})$ muons is higher in this sample. In addition, cosmic ray muons also enter the detector from above.

In an earlier paper [3], the response of ICAL to few-GeV muons with respect to both momentum magnitude and direction reconstruction was studied through simulations for muons generated in the central region of ICAL where the magnetic field is largely uniform both in direction and magnitude. Here we present for the first time a GEANT4-based simulations study of the muon response in the peripheral region of ICAL, where the magnetic field is not only highly non-uniform in both magnitude and direction, but there are significant edge effects as most of the tracks will be partially contained. Note that a substantial fraction of rock muon events traverses such regions in the detector; hence it is important to understand the muon response in these regions for such physics studies.

The inclusion of muon response in the peripheral region of the detector can significantly change the oscillation and mass hierarchy results since it contains resolutions and efficiencies in the energy range $1-50 \mathrm{GeV}$. The first set of simulations results for precision measurement of neutrino oscillation parameters and the mass hierarchy were produced using only the central region resolutions of about $9-14 \%$ and efficiency of about $80 \%$ (see Ref. [4], [5] for more details) in the energy range $1-20 \mathrm{GeV}$.

The paper is organised as follows: in Section 2 we briefly discuss some relevant details about the GEANT4-based simulation of the detector geometry and magnetic field, as well as the methodology of hit and track generation and track reconstruction. In Section 3, we discuss the general features of muon propagation in the different regions of ICAL. In Section 4, we discuss the selection criteria used. We then present in Section 5 the results, with these 
selection criteria, for the muon efficiencies and resolutions in the peripheral region of ICAL. A comparison of the response in all the regions of ICAL (central and peripheral) is given in Section 6. We conclude in Section 7, with discussions.

\section{The ICAL Detector Simulation Framework}

Details of the ICAL detector can be found elsewhere [3]. Here we briefly review the relevant simulation inputs for the geometry and magnetic field. The ICAL detector geometry is simulated [3] using GEANT4 software [6]. It comprises of three identical modules of dimension 16 $\mathrm{m} \times 16 \mathrm{~m} \times 14.45 \mathrm{~m}$. The direction along which the modules are placed is chosen to be the $x$-direction (and is labelled with the azimuthal angle $\phi=0$ ) and the direction perpendicular to the $x$-axis in the horizontal plane is the $y$-direction. The vertical direction is the $z$-direction with the $z$-axis pointing upwards (so the polar angle is also the zenith angle). Each module comprises a stack of 151 layers of $5.6 \mathrm{~cm}$ thick magnetized iron, separated by a $4 \mathrm{~cm}$ air gap in which the active detector elements, the RPCs are placed. The $y$-direction is in the plane of the iron plates, parallel to the slots of the magnet coil, as shown in Fig. 1, with the origin of the coordinate system being the centre of the central module.

Apart from coil slots (thin $8 \mathrm{~m}$ long slots centred around $y=0$ at $x=x_{0} \pm 4 \mathrm{~m}$, where $x_{0}$ is the central $x$-coordinate of each module), there are vertical steel support structures which are placed at every $2 \mathrm{~m}$ along the whole detector in both $x$ and $y$ directions, to maintain the air gap between plates. These are dead spaces that affect the muon reconstruction. In addition, the magnetic field is also not uniform everywhere and so the quality of reconstruction depends on the region where the event is located.

\subsection{The Magnetic Field}

The magnetic field has been simulated in a single iron plate using the MAGNET6 software [7. The magnetic field map is generated at the centre $(z=0)$ of the plate and is assumed uniform over the entire thickness of the plate. The magnetic field lines in a single iron plate in the central module are shown in Fig. 1. The field is generated by current circulating in copper coils that pass through slots in the magnetized plates. The slots can be seen as thin white vertical lines at $x= \pm 4 \mathrm{~m}$ in the figure. The direction and length of arrows denote the direction and magnitude of the magnetic field.

The "central region" is defined as the volume contained within the region $|x| \leq 4 \mathrm{~m},|y| \leq 4$ $\mathrm{m}$ with $z$ unconstrained, that is, the region within the coils slots in each module. The central region has the highest magnetic field that is uniform in both magnitude and direction $\left(B_{y}\right)$ while the region labelled "peripheral region" (outside the central region in the $y$ direction, $|y|>4 \mathrm{~m}$ ) has the maximally changing magnetic field in both direction and magnitude, with the field falling to zero at the corners of the module.

In an earlier paper [3], the muon response was studied in the central region; here we study the peripheral region where, apart from the changing magnetic field, the reconstruction is also affected by edge effects. In addition, there are two small regions outside the coil slots in the $x$ direction $(|x|>4 \mathrm{~m})$, labelled as the "side region" in Fig. 1 where the magnetic field is about $15 \%$ smaller than in the central region and in the opposite direction. The side regions in the central module are contiguous with the side regions in the adjacent modules and the quality of reconstruction here is expected to be similar to that in the central region. However the left 


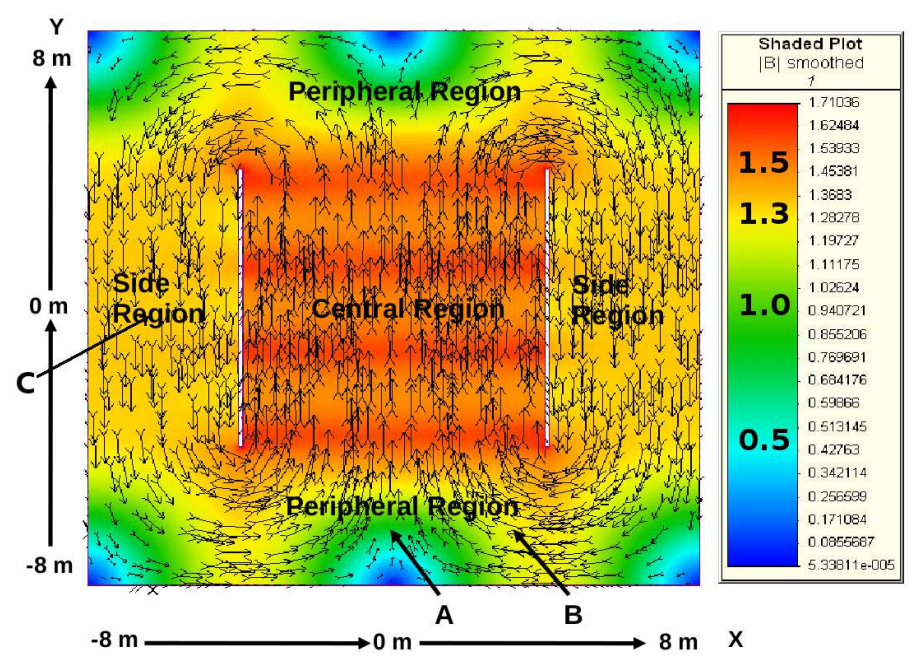

Fig. 1: Magnetic field map as generated by the MAGNET6 [7] software in the central iron layer of the central module. Points $\mathrm{A}=(0,-650,0) \mathrm{cm}, \mathrm{B}=(300,-650,0) \mathrm{cm}, \mathrm{C}=(-2270,0,0)$ $\mathrm{cm}$ (in the $1^{\text {st }}$ module of ICAL), are defined for later use. Notice that $\mathrm{C}$ is actually in the left-most module of the detector and is simply marked here for convenience.

(right) side region of the left (right)-most module will suffer from edge effects and we shall consider them separately in the study.

\subsection{Event Reconstruction}

In each analysis, 10,000 muons are propagated in the detector with fixed momenta and direction and with the starting point of the muons in either the peripheral or side regions. In contrast to the earlier study in the central region [3], here the muon momenta vary from 1-50 $\mathrm{GeV} / \mathrm{c}$, with the higher energies being of interest for rock muon studies. The muon tracks are reconstructed based on a Kalman filter [8] algorithm. The RPCs that signal the passage of muons through them have a position sensitivity of about $\pm 1 \mathrm{~cm}$ in the $x$-and $y$-directions (the $\mathrm{RPC}$ strip width is $1.96 \mathrm{~cm}$ ) and about $\pm 1 \mathrm{~mm}$ in the $z$-direction (the gas gap in the RPCs being $2 \mathrm{~mm}$ ). In addition, "hits" or signals can be generated in adjoining RPC strips as well, and further that the RPC efficiency and time resolution are about $95 \%$ and 1 ns respectively 9]. For more details regarding the generation of hits, tracks and their reconstruction, see Ref. [3].

\section{General Feature of Muon Response in the Peripheral and Side Regions}

We first discuss the general expectations, based on the detector geometry and the orientation of the magnetic field.

The Lorentz force equations are $\vec{F}=q(\vec{v} \times \vec{B})$, where $\vec{B}$ is the magnetic field that is confined to the $x-y$ plane, $q$ is the charge of the particle with momentum $\vec{P}$ and energy $E$ so that its velocity $\vec{v}$ is directed along the momentum with magnitude $v=P c^{2} / E$. Since 
$q=-1$ for $\mu^{-}$, the components of force in the peripheral region for an upward-going muon, momentarily ignoring energy loss, are,

$$
\begin{aligned}
& F_{x}=v_{z} B_{y} \\
& F_{y}=-v_{z} B_{x} \\
& F_{z}=v_{y} B_{x}-v_{x} B_{y}
\end{aligned}
$$

whereas the analogous components of force in the side region are given as:

$$
\begin{aligned}
& F_{x}=-v_{z} B_{y} ; \\
& F_{y}=0 ; \\
& F_{z}=v_{x} B_{y} .
\end{aligned}
$$

It is seen that in both regions, $F_{x}$ and $F_{y}$ are independent of $\phi$ (that is, independent of the momentum components in the plane of the field) and depend on $P_{z}$ (i.e., on the zenith angle $\theta$ alone) while $F_{z}$ depends on both $\theta$ and $\phi$. Depending on the components of magnetic field $B_{x}$ and $B_{y}$, Eqs. 1 and 2 give the net force in the different regions of ICAL. Consider the in-plane ( $x, y$ components) forces in the regions denoted as 1-10 in Fig. 2. It can be seen that in regions $1,2,7,8, F_{y}$ is such that it causes an upward-going muon $(\cos \theta>0)$ to be bent in a direction going out of the detector, thus lowering the reconstruction efficiency. The effect is just the opposite in regions $3,4,5,6$. If $F_{z}>0$ as well, the already upward-going muon traverses more iron layers as discussed in Ref. [3] and hence gives good resolution; hence, $F_{y}$ affects the reconstruction efficiency while $F_{z}$ determines the quality of reconstruction. Since $F_{z}$ depends on both $(\theta, \phi)$ as well as the magnetic field, the sign of $F_{z}$ is shown in Fig. 2 inside a circle of $\phi$ in each of regions 1,2,3,4 (for negatively charged upward-going muons), for $\left|B_{x}\right| \sim\left|B_{y}\right|$ with purple (cyan) regions denoting $F_{z}>(<) 0$.

Hence the magnetic field that determines the quality of reconstruction breaks the azimuthal symmetry, so muons in different $\phi$ directions (for the same momenta and polar angle $\cos \theta$ ) have different detector response. This was discussed in detail in Ref. [3]. Going by the force equations, we therefore analyse the muon response in the peripheral region in four different set of $\phi$ bins as shown in Fig. 3: bin I: $|\phi| \leq \pi / 4$, bin II: $\pi / 4 \leq \phi<3 \pi / 4$, bin III: $-3 \pi / 4 \leq \phi<-\pi / 4$, and bin IV: $3 \pi / 4<|\phi| \leq \pi$.

For muons with starting point in the negative $y$ peripheral region (regions marked 1, 2, 3, 4 in Fig. 2), this implies that most of the muons with momenta such that $\phi$ lies in bin III (but otherwise having same magnitude and $\cos \theta$ ) are prone to exit the detector from the side; however, there will be marked differences in the quality of reconstruction between regions $(1,2)$ and $(3,4)$ due to the different $F_{y}$ force that turns the track back into the detector in regions 3,4 . Hence the average detector response in this region is an average over these two different behaviours. In addition, $F_{z}>0(<0)$ for bin II muons in regions $1,2(3,4)$ and this helps to improve the reconstruction in regions 1,2 , so that bin II muons can be expected to have the best quality of reconstruction of the regions 1-4.

A similar analysis can be done for muons which begin in the positive $y$ peripheral region (regions marked 5,6,7,8). Of course, tracks at the edge of a region or of high energy muons may move from one region to another where a different magnetic field applies; however, we simply bin the events according to the region in which the muon originates. 


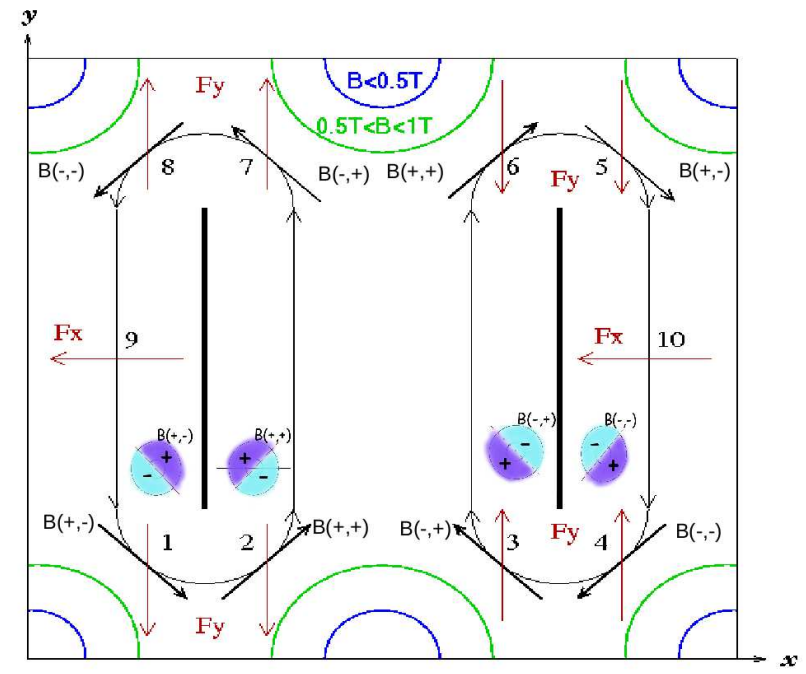

Fig. 2: Magnetic field map with the net force directions in the peripheral and side region. The thick black arrows indicate the direction of the magnetic field with labels $B(i, j), i=+,-$, that denote the sign of the $B_{x}, B_{y}$ components in each region. The brown arrows indicate the direction of $F_{x}$ or $F_{y}$ force components that will act on a negatively charged upward-going muon. The small coloured circles indicate the direction of $F_{z}$ in each region, with purple (cyan) denoting $F_{z}>0(<0)$. (Note that side regions 9 and 10 are in the $1^{\text {st }}$ and $3^{\text {rd }}$ modules of the detector respectively and are shown together in the same module for convenience.)
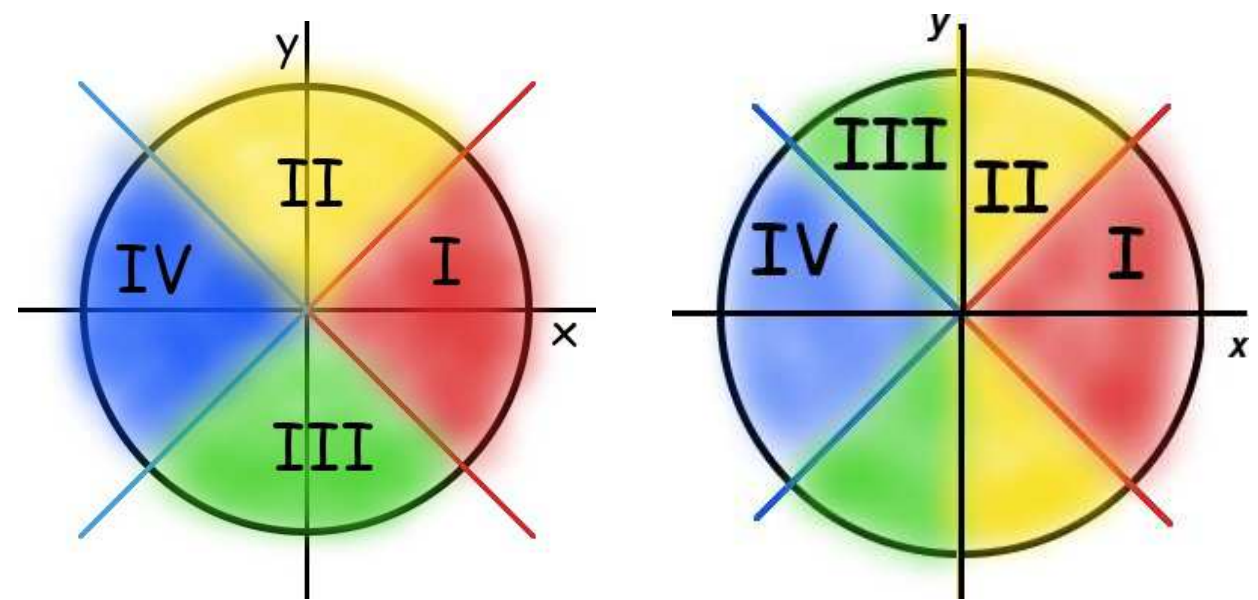

Fig. 3: The choice of $\phi$ bins in the peripheral (left) and side (right) regions. 
In the side region $9, F_{x}$ causes the particle to go out of the detector but $F_{z}>0$ and so this region has good resolution but worse efficiency. The results are opposite in side region 10 since $B_{y}<0$. We therefore define the same $\phi$ bins for the side region as were used for the central region, viz., bin I: $|\phi| \leq \pi / 4$, bin II: $\pi / 4<|\phi| \leq \pi / 2$, bin III: $\pi / 2<|\phi| \leq 3 \pi / 4$, and bin IV: $3 \pi / 4<|\phi| \leq \pi$. The difference is in the definition of the second and third bins, see Fig. 3, and is more appropriate from the point of view of the geometrical configuration in this region. Region 9 (10) will have the worst reconstruction in $\phi$ bin IV (I). However, in region 10 , the direction of the $F_{x}$ force is expected to improve the results just as in the case of regions 3,4 .

The results will be the same for downward-coming $\mu^{+}$(with $\cos \theta<0$ ) while that for downward-coming muons or upward-going anti-muons can be obtained by symmetry. In our analysis, therefore, we study the muon response in the peripheral regions $1-4$ and the side region 9.

It is important to keep in mind that the support structures and coil gaps also break this azimuthal symmetry in a non-trivial way and the effects of the geometry may alter the trends of the distributions as discussed above.

The net effect of the nature of the detector geometry and magnetic field can be seen in the muon resolutions and efficiencies and we will now discuss these.

As discussed above, we study the response in the following peripheral and side regions.

In the Peripheral Region : Here, 10,000 muons $\left(\mu^{-}\right)$were propagated with fixed input momenta $P_{\text {in }}$ and direction $\cos \theta$ (and smeared over the entire azimuthal angle $\phi$ ), with their starting point uniformly smeared over the region centred at $(0,-600,0) \mathrm{cm}$ and extending upto $\pm(2400,200,720) \mathrm{cm}$ from it; this comprises the whole peripheral region along the three modules of the detector in the negative $y$ region where the magnetic field is non-uniform.

In the Side Region : In the side region, muons $\left(\mu^{-}\right)$were propagated with the same procedure as above but with point of origin smeared in a region centred around $(-2200,0,0)$ $\mathrm{cm}$ and $(2200,0,0) \mathrm{cm}$ (which are in the $1^{\text {st }}$ and $3^{\text {rd }}$ modules of the detector respectively) and smeared uniformly in $\pm(200,400,720)$ cm around these.

\section{Selection Criteria Used}

All tracks which satisfy the loose selection criterion $\chi^{2} / \mathrm{ndf} \leq 10$, are used in the analysis, where $\chi^{2}$ is the standard $\chi$-squared of the fit and ndf are the number of degrees of freedom, ndf $=2 \times N_{\text {hits }}-5$, where $N_{\text {hits }}$ are the number of hits in the event, $N_{\text {hits }} \geq 5$ and the Kalman filter involves the fitting of 5 parameters.

Further selection criteria are used to get reasonable fits and hence resolutions. Two major constraints have been applied in both the peripheral and side regions to remove low energy tails. The first is similar to that applied when analysing tracks in the central region [3]: the Kalman filter algorithm may generate more than one track. While this may be correct and useful in the case of genuine neutrino CC interactions, where one or more hadrons accompany the muon, this is a problem for single muon analysis and arises because of detector dead spaces (for instance, two portions of a track on either side of a support structure may be reconstructed as two different tracks). This problem will be mitigated by the identification 
of a vertex in a genuine neutrino interaction, here, we place a constraint and analyse only those events for which exactly one track is reconstructed, leading to a consequent loss in reconstruction efficiency. The second selection criterion is specific to the peripheral and side regions and is described below.

Initially, events were generated at fixed points of origin to understand the effect of the magnetic field. In the peripheral region, the starting point was chosen to be either at point A (in a region of nearly zero magnetic field) or B (large magnetic field with both $x$ - and $y$-components non-zero), while in the side region, a generic point $\mathrm{C}$ was studied (see Fig. 11). Results of this study clearly indicated that a large fraction of events whose tracks were truncated because the particle exited the detector (so-called partially contained events) were relatively poorly reconstructed. These could not be eliminated by tightening the constraint on $\chi^{2}$ of the fits; however, they could largely be removed by demanding that the track contain a minimum number of hits, such that either $N_{\text {hits }}>n_{0}$ or $N_{\text {hits }} / \cos \theta>n_{0}$ (note that there may be multiple hits per layer), where $n_{0}$ needed to be carefully optimised. It was found that for a given momentum and direction of the muon, $n_{0}$ needed to be larger (smaller) in regions where the magnetic field strength is small (large). Where the muon does not leave the detector, so that the entire track is contained in the detector (fully contained events), no constraint on $N_{\text {hits }}$ is needed. With this understanding, the generic peripheral and side region response was studied.

\subsection{Effect of Selection Criteria}

The effect of $N_{\text {hits }}>n_{0}$ or $N_{\text {hits }} / \cos \theta>n_{0}$ can be seen from Fig. 4 for the peripheral region. If the event is fully contained, there is no constraint on $N_{\text {hits }}$; the effect of $n_{0}=15$ is shown in the left-hand side of Fig. 4 for $\left(P_{i n}, \cos \theta\right)=(5 \mathrm{GeV} / \mathrm{c}, 0.65)$ and $(9 \mathrm{GeV} / \mathrm{c}, 0.85)$ where the histogram in the magnitude of the reconstructed momentum $P_{\text {rec }}$ is plotted. For $P_{\text {in }}=5$ $\mathrm{GeV} / \mathrm{c}$, it is noticed that the $N_{\text {hits }}$ constraint does not affect the $P_{\text {rec }}$ momentum distribution much, as most of the events are fully contained. But it gives a better (more symmetrical) shape to the distribution by reducing the low-energy tail. On the other hand, the effect for $P_{\text {in }}=9$ $\mathrm{GeV} / \mathrm{c}$ is stronger, with the hump at lower $P_{\text {rec }}$ being eliminated with the $N_{\text {hits }}$ selection criteria. Fig. 5 shows the effect of $N_{\text {hits }}>n_{0}$ or $N_{\text {hits }} / \cos \theta>n_{0}$ on $N_{\text {hits }}$ distributions in the peripheral region. In all cases, the few events surviving below the constraint are from totally contained events, on which no constraint is placed. These cause the histograms to remain non-zero in the region $N_{\text {hits }} \leq n_{0}$ or $N_{\text {hits }} / \cos \theta \leq n_{0}$ as seen in Fig. 5. However, these events are relatively few in number, being less than $2 \%(3 \%)$ of the total reconstructed events for $n_{0}=15$ (20) in Fig. 5. The constraint $N_{\text {hits }} / \cos \theta>15$ is the most conservative one, with a loss of only about $10 \%$ of the reconstructed events and is found to be more optimal than $N_{\text {hits }}>n_{0}$.

Different choices of $n_{0}$ can be used. We have shown the effect of (a) no constraint, (b) $N_{\text {hits }}>15$, and (c) $N_{\text {hits }} / \cos \theta>15$ in the left-hand side of Fig. 4 . The last choice is motivated by the fact that a slant-moving muon (in the absence of magnetic field) would move a distance $d / \cos \theta$ in comparison to a vertically upward-going muon of the same momentum that would traverse a distance $d$. Similar figures on the right use the choice $n_{0}=20$, with the more stringent requirement giving distributions with correspondingly smaller root-mean-square or square root of the variance (RMS widths) by about 7-8\%, but showing a decrease in the total number of reconstructed events by 10-15\%. Note also that increasing $n_{0}$ eventually leads to 

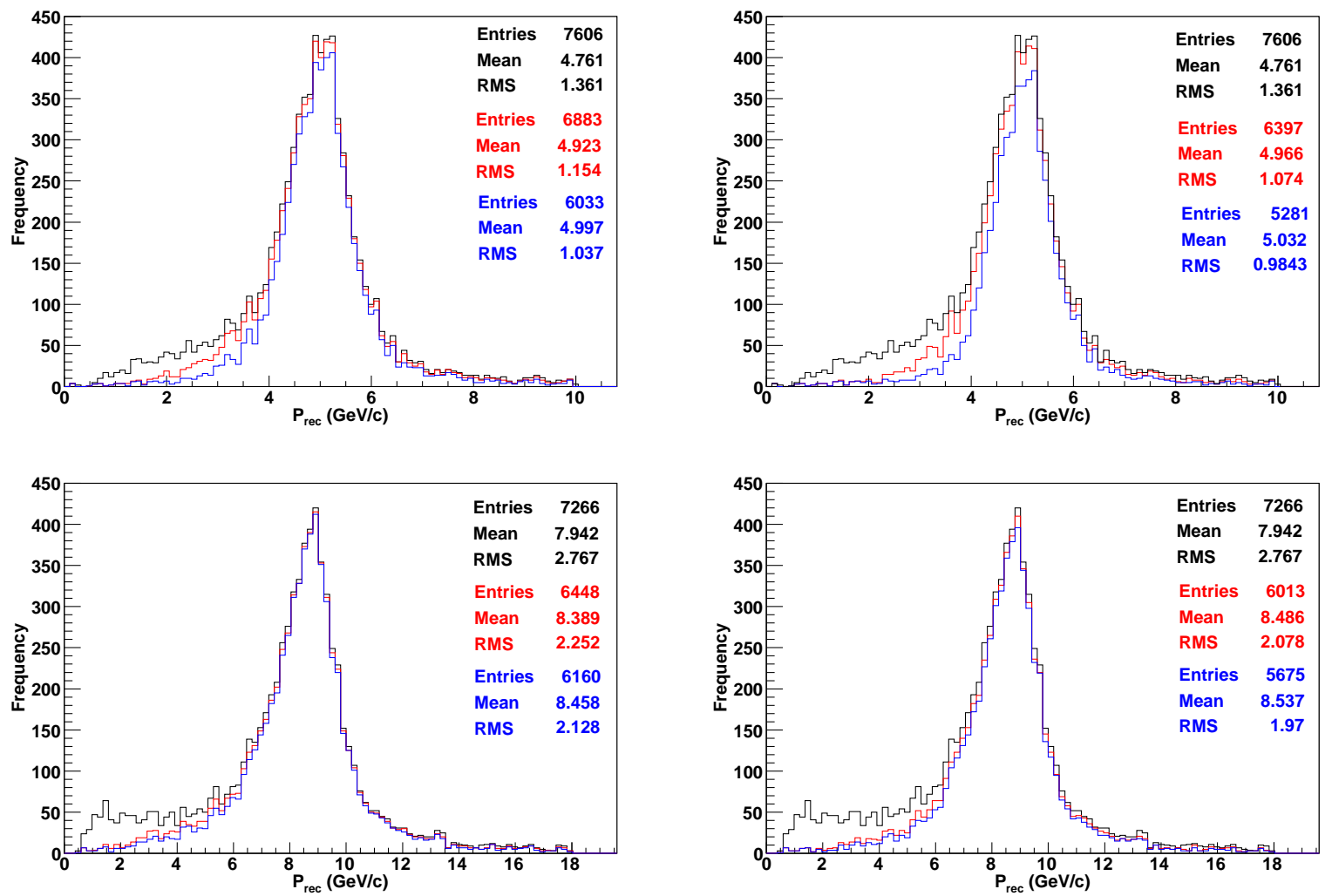

Fig. 4: Top (bottom) figures show the reconstructed momenta $P_{\text {rec }}$ using selection criteria $N_{\text {hits }}>n_{0}$ for partially contained events in the peripheral region with $\left(P_{\text {in }}, \cos \theta\right)=(5$ $\mathrm{GeV} / \mathrm{c}, 0.65)$ (top) and $(9 \mathrm{GeV} / \mathrm{c}, 0.85)$ (bottom) with $n_{0}=15$ (20) in the left (right) figure. Fully contained events have no $N_{\text {hits }}$ constraint. In each figure, the black curve is without constraints on $N_{\text {hits }}$, red is with $N_{\text {hits }} / \cos \theta>n_{0}$ and blue is for $N_{\text {hits }}>n_{0}$. 

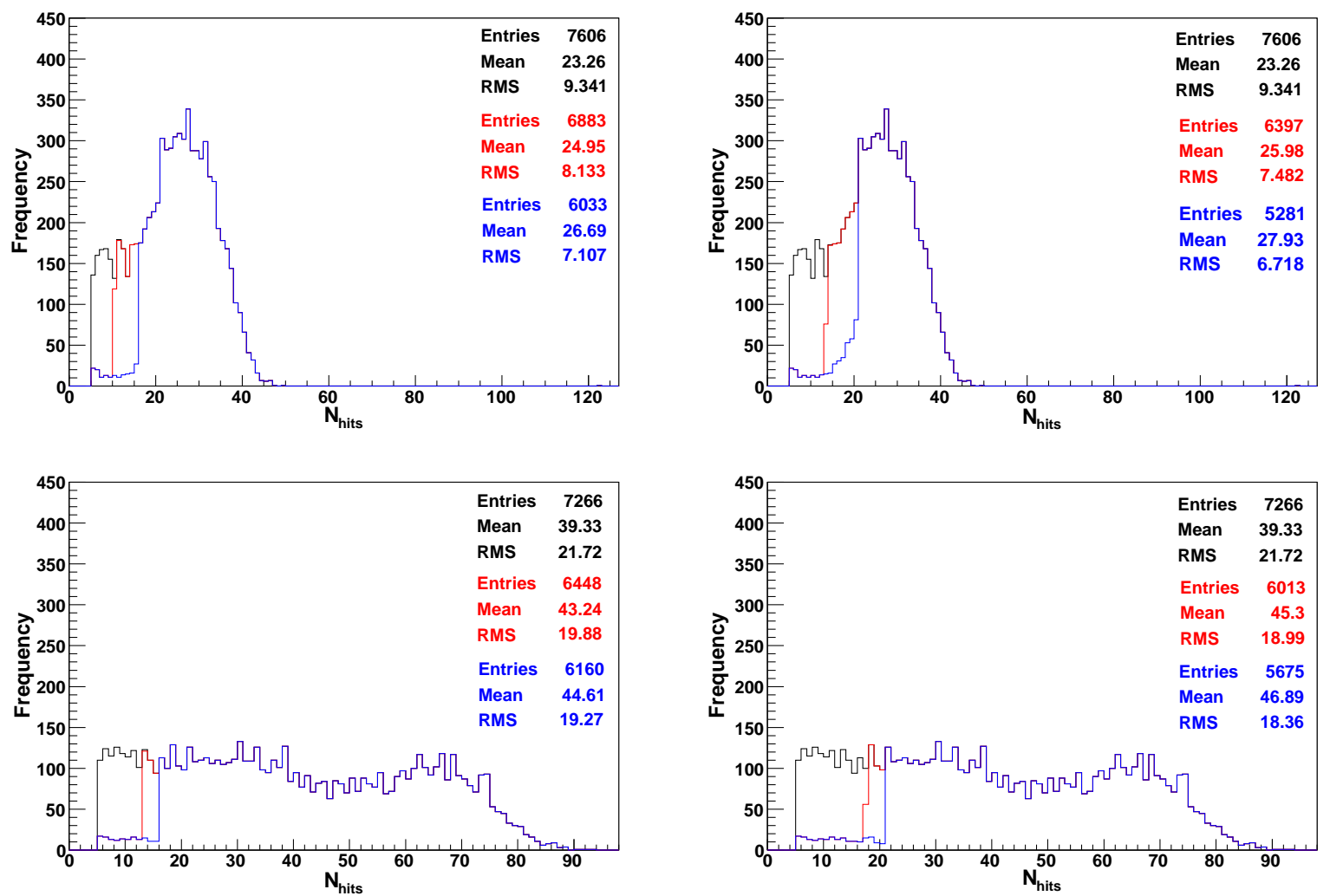

Fig. 5: Top (bottom) figures show the $N_{\text {hits }}$ distributions using selection criteria $N_{\text {hits }}>n_{0}$ for partially contained events in the peripheral region with $\left(P_{\text {in }}, \cos \theta\right)=(5 \mathrm{GeV} / \mathrm{c}, 0.65)$ (top) and $(9 \mathrm{GeV} / \mathrm{c}, 0.85)$ (bottom) with $n_{0}=15$ (20) in the left (right) figure. Fully contained events have no $N_{\text {hits }}$ constraint. In each figure, the black curve is without constraints on $N_{\text {hits }}$, red is with $N_{\text {hits }} / \cos \theta>n_{0}$ and blue is for $N_{\text {hits }}>n_{0}$. 
removal of well-reconstructed events, as visible from the loss of events in the peak apart from just trimming the tails for the lower momentum $P_{i n}=5 \mathrm{GeV} / \mathrm{c}$ for $n_{0}=20$.

Similarly, the effect of the selection criteria on the reconstruction in the side regions is shown in Fig. 6. Here the constraint on the partially contained events is not as strongly marked as in the peripheral region: while there is certainly a decrease in the RMS width of the distribution and in the number of selected events when the constraint is applied, a larger fraction of events are lost due to the constraint, with a number of "good" events being lost from the peak of the distribution as well, unlike in the peripheral region.
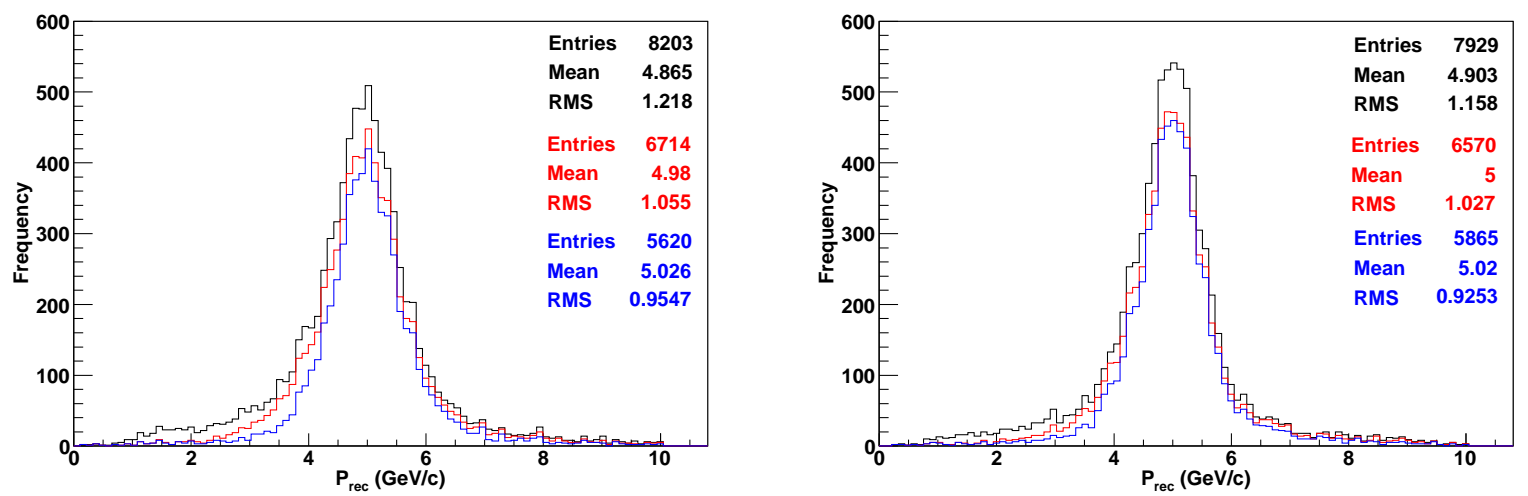

Fig. 6: The figures show the reconstructed momenta $P_{\text {rec }}$ using the selection criteria $N_{\text {hits }}>n_{0}$ for partially contained events in the side regions 9 (left) and 10 (right) for $\left(P_{\text {in }}, \cos \theta\right)=(5$ $\mathrm{GeV} / \mathrm{c}, 0.65)$ with $n_{0}=15$. Fully contained events have no $N_{\text {hits }}$ constraint. In each figure, the black curve is without constraints on $N_{\text {hits }}$, red is with $N_{\text {hits }} / \cos \theta>n_{0}$ and blue is for $N_{\text {hits }}>n_{0}$.

The final choice of selection criteria will be guided by the physics study. In case the requirement is good momentum resolution, then the choice $n_{0}=20$ may be appropriate (that is, either $N_{\text {hits }}>20$ or $N_{\text {hits }} / \cos \theta>20$ ). However, since the shape of the distribution is already reasonable for $n_{0}=15$, this choice may be used when the focus is not so much on precision reconstruction but on higher event reconstruction rates. In the rest of this paper, we shall apply the constraint $N_{\text {hits }} / \cos \theta>15$ as being appropriate and sufficient. This choice also improves the reconstruction efficiency of large angle (small $\cos \theta)$ events whose tracks naturally contain fewer hits and are harder to reconstruct.

In the next section, we present the results on muon resolution and efficiencies in the peripheral and side region using these selection criteria.

\section{Muon Response in the Peripheral and Side Regions}

\subsection{Momentum Reconstruction Efficiency}

The reconstruction efficiency is defined as the ratio of the number of reconstructed events $n_{\text {rec }}$ (irrespective of charge) to the total number of events, $N_{\text {total }}$. We have

$$
\begin{aligned}
\epsilon_{\text {rec }} & =\frac{n_{\text {rec }}}{N_{\text {total }}}, \\
\text { with error } \delta \epsilon_{\text {rec }} & =\sqrt{\epsilon_{\text {rec }}\left(1-\epsilon_{\text {rec }}\right) / N_{\text {total }}} .
\end{aligned}
$$


Fig. 7 shows the reconstruction efficiency averaged over $\phi$ as a function of input momentum for different $\cos \theta$ values in the peripheral and side regions.
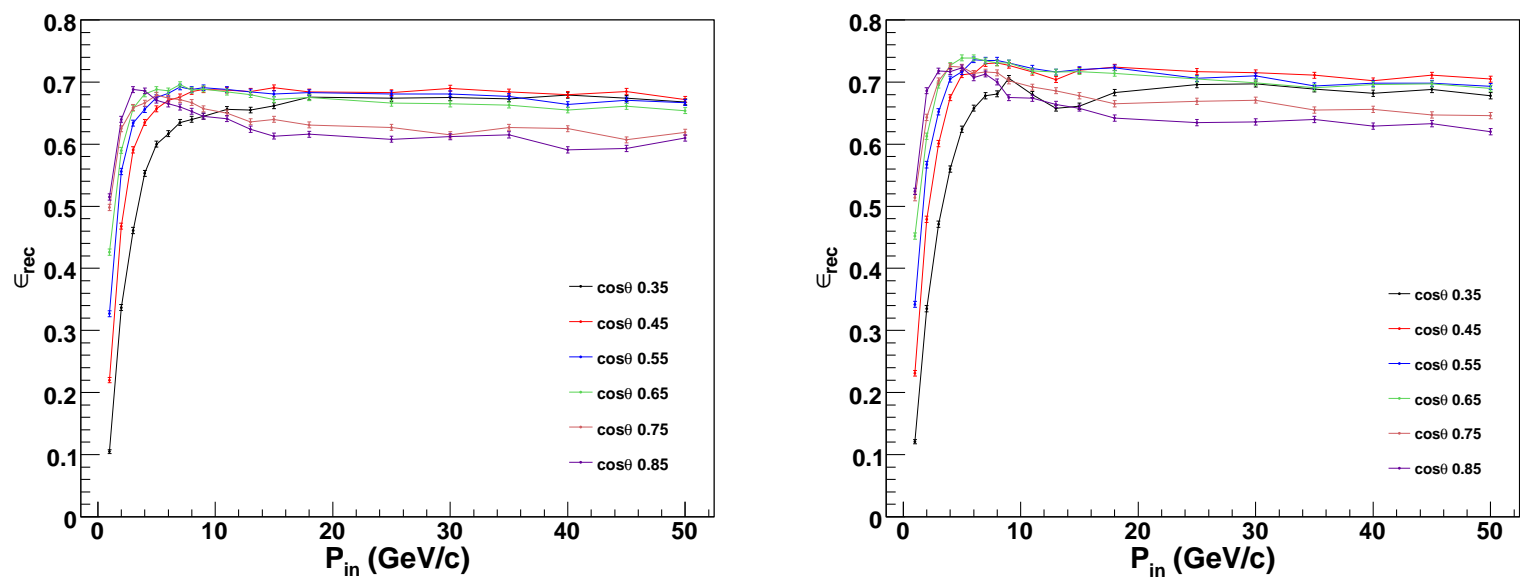

Fig. 7: Reconstruction efficiency averaged over all $\phi$ bins as a function of the input momentum $P_{\text {in }}(\mathrm{GeV} / \mathrm{c})$ for different zenith angles $\cos \theta$ in the peripheral (left) and side 9 (right) regions. For a discussion of the selection criteria see the text.

The reconstruction efficiency increases for all angles, from $P_{i n}=1 \mathrm{GeV} / \mathrm{c}$ since the number of hits increases as the particle crosses more layers. Since there are fewer hits for more slantangled muons, the efficiency at a given momentum is better for larger values of $\cos \theta$. Also, the reconstruction efficiency is very similar for all the peripheral and side regions.

In all cases, the slight worsening of the efficiency for $\cos \theta=0.85$ at higher momenta is spurious and is due to the selection criterion that the event should reconstruct exactly one track. At large angles, it is more likely that two portions of a track on either side of a dead space such as a support structure are reconstructed as two separate tracks. Efforts are on to retrieve such events by improving the reconstruction code [10]. When these tracks are correctly reconstructed, the efficiency is expected to saturate rather than fall off at these momentum values. Again, such tracks are not expected to be troublesome in genuine neutrino events, as discussed earlier.

\subsection{Relative Charge Identification Efficiency}

The charge identification (cid) of the particle is critical in many studies since it distinguishes events initiated by neutrinos and anti-neutrinos; these have different matter effects as they propagate through the Earth and hence give the required sensitivity to the neutrino mass hierarchy. The charge of the particle is determined from the direction of curvature of the track in the magnetic field. Relative charge identification efficiency is defined as the ratio of the number of events with correct charge identification, $n_{\text {cid }}$, to the total number of reconstructed events, $n_{\text {rec }}$, i.e.,

$$
\epsilon_{\mathrm{cid}}=\frac{n_{\mathrm{cid}}}{n_{\mathrm{rec}}},
$$

where the errors in $n_{\text {cid }}$ and $n_{\text {rec }}$ are correlated so that the error in the ratio is calculated as:

$$
\delta \epsilon_{\text {cid }}=\sqrt{\epsilon_{\text {cid }}\left(1-\epsilon_{\text {cid }}\right) / n_{\text {rec }}} .
$$


Fig. 8 shows the relative charge identification efficiency as a function of input momentum for different $\cos \theta$ values in the peripheral and side region 9. (Similar results apply for side region 10). The muon undergoes multiple scattering while propagating in the detector; for small momentum, since the number of layers traversed is small, this may lead to an incorrectly reconstructed direction of bending, resulting in the wrong charge identification. Hence the charge identification efficiency is relatively poor at lower energies but as the energy increases cid efficiency also improves. At very high input momenta, bending due to the magnetic field is less. For partially contained events, only the initial relatively straight portion of the track is contained within the detector; this leads to large momentum uncertainty as well as mis-identification of charge. Overall the relative charge identification efficiency is marginally smaller than in the central region because of the smaller magnetic field.
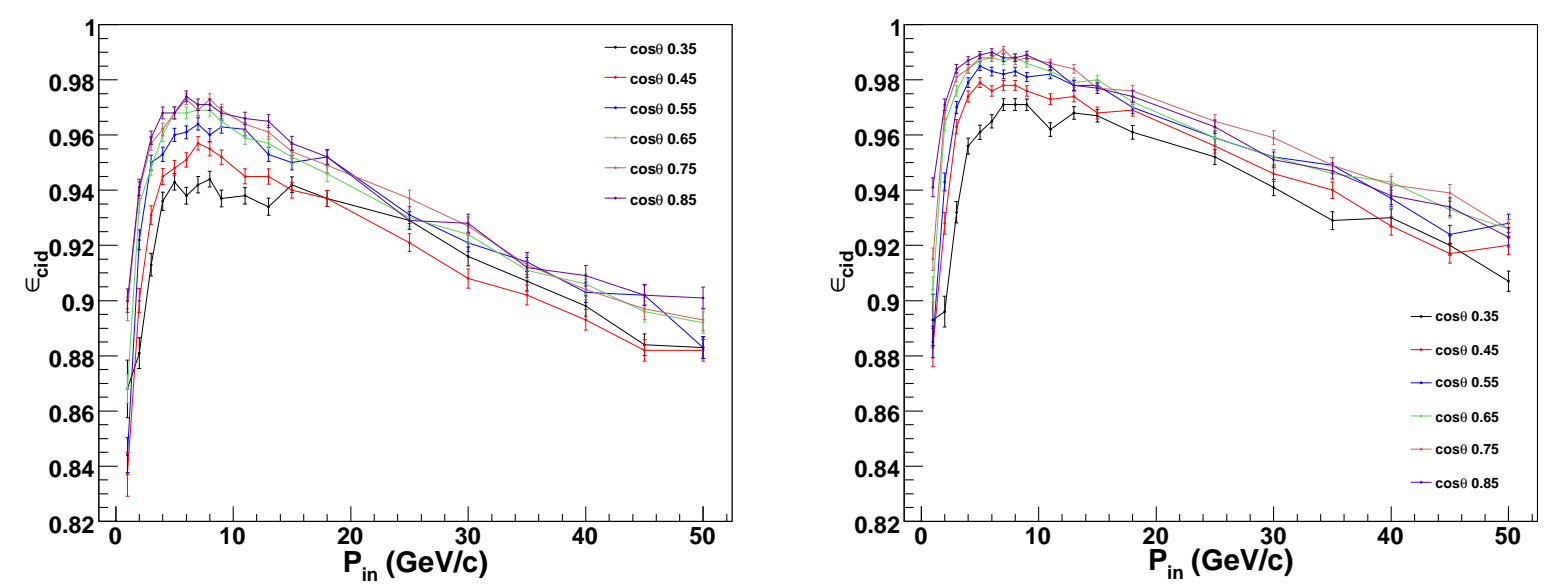

Fig. 8: Charge identification (cid) efficiency averaged over all $\phi$ bins as a function of the input momentum $P_{\text {in }}(\mathrm{GeV} / \mathrm{c})$ for different zenith angles $\cos \theta$ in the peripheral (left) and side 9 (right) regions. For a discussion of the selection criteria see the text.

\subsection{Direction (up/down) Reconstruction}

The reconstructed zenith angle distributions for $P_{i n}=1 \mathrm{GeV} / \mathrm{c}$ at $\cos \theta=0.35$ and $\cos \theta=$ 0.85 in the peripheral and side region 9 are shown in Figs. 9 and 10 respectively.

From Fig. 9, it is noticed that there are few events reconstructed in the downward direction (wrong direction) with $\theta_{\text {rec }}>\pi / 2$. For $P_{\text {in }}=1 \mathrm{GeV} / \mathrm{c}$ with $\cos \theta=0.35$ (0.85), this fraction is about $0.48(0.89) \%$ and it drops to a negligible value at higher energies for all $\cos \theta$. Similar results are obtained for the side region 9 as can be seen from Fig. 10. This small fraction also contributes to wrong cid since the relative bending in the magnetic field is measured w.r.t the muon momentum direction.

The direction determination depends on the time resolution while the charge identification depends also on the strength of the magnetic field. A $1 \mathrm{GeV} / \mathrm{c}$ muon with $\cos \theta \sim 1$ traverses about 12 layers; this corresponds to a time difference between first and last hit of about $4 \mathrm{~ns}$. Since the RPCs have a time resolution of $1 \mathrm{~ns}$, this explains why the fraction of muons whose direction is wrongly determined is small. 

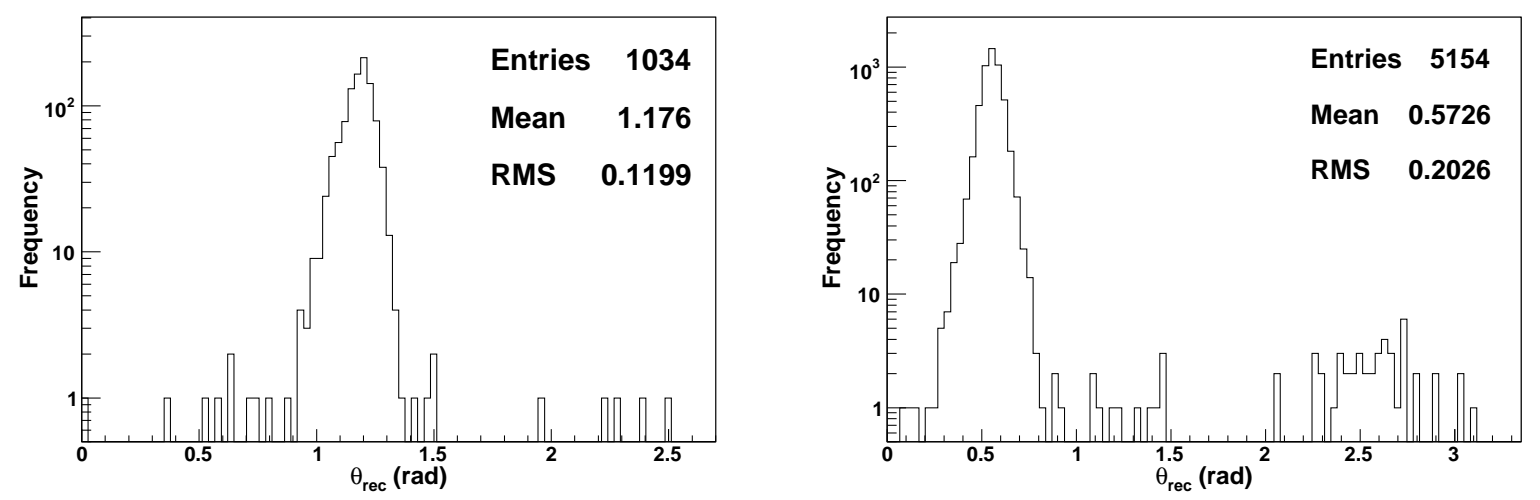

Fig. 9: Reconstructed zenith angle distributions for $P_{\text {in }}=1 \mathrm{GeV} / \mathrm{c}$ at $\cos \theta=0.35$ (left) and 0.85 (right) respectively, in the peripheral region. Note that the $y$-axis scales are different for the two plots.
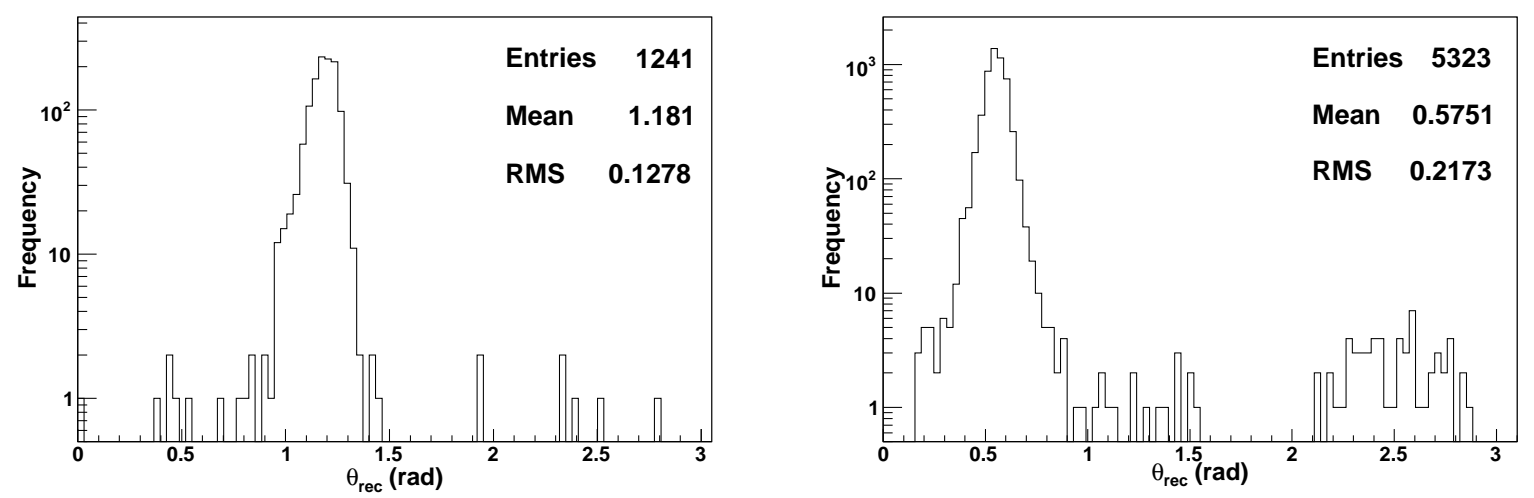

Fig. 10: Reconstructed zenith angle distributions for $P_{i n}=1 \mathrm{GeV} / \mathrm{c}$ at $\cos \theta=0.35$ (left) and 0.85 (right) respectively, in the side region 9 . Note that the y-axis scales are different for the two plots. 


\subsection{Zenith Angle Resolution}

Those events which are successfully reconstructed (for all $\phi$ ) are analysed for their zenith angle resolution. The events distribution as a function of the reconstructed zenith angle $\theta_{\text {rec }}$ is shown in Fig. 11 for a sample input $\left(P_{\text {in }}, \cos \theta\right)=(5 \mathrm{GeV} / \mathrm{c}, 0.65)$ for the peripheral and side region 9 respectively.
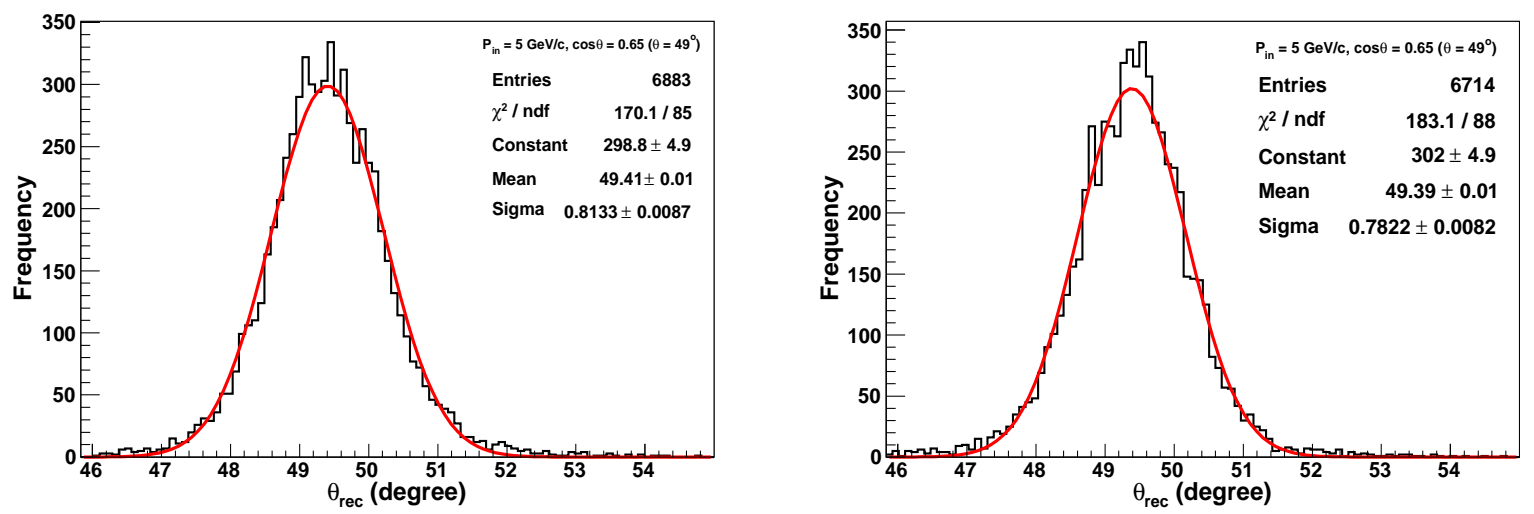

Fig. 11: Reconstructed distribution $\theta_{\text {rec }}$ for input $\left(P_{\text {in }}, \cos \theta\right)=(5 \mathrm{GeV} / \mathrm{c}, 0.65)$ in the peripheral region (left) and side region 9 (right). The selection criteria are the same as before.

The angular resolution is good in both the regions and is in fact better than about a degree for input momentum greater than a few $\mathrm{GeV}$, as seen in Fig. 12, with the resolution being marginally better in the side region. Similar results are obtained in side region 10 as well. In addition, the fraction of events reconstructed in the wrong direction (wrong quadrant of $\cos \theta)$ is negligibly small, being less than $0.5 \%$ for $P_{\text {in }} \geq 2 \mathrm{GeV} / \mathrm{c}$.
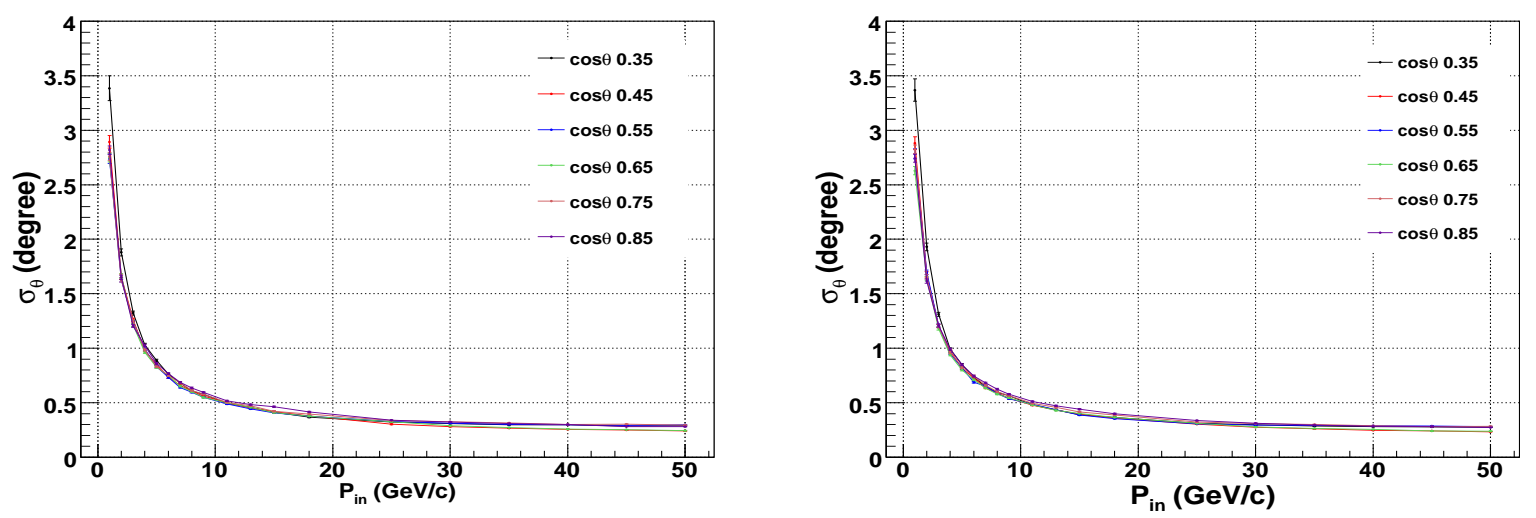

Fig. 12: Resolution, $\sigma_{\theta}$, of reconstructed angle $\theta_{\text {rec }}$ as a function of the input momentum $P_{\text {in }}$ $(\mathrm{GeV} / \mathrm{c})$ for different values of input $\cos \theta$ in the peripheral region (left) and side region 9 (right). The selection criteria are the same as before. 


\subsection{Muon Momentum Response}

While the cid efficiency and zenith angle resolution are insensitive to the azimuthal angle $\phi$, due to the reasons given above, we analyse the muon momentum response in different $\phi$ bins. The response is shown in Fig. 13 for the peripheral region with the constraint $N_{\text {hits }} / \cos \theta>15$ being applied as usual to the partially contained events, for sample input values of $\left(P_{i n}, \cos \theta\right)=(5 \mathrm{GeV} / \mathrm{c}, 0.65)$.

The histograms in $P_{\text {rec }}$ have been fitted with Gaussian functions. The width of each distribution of the four sets differs while the mean remains similar. As expected, $\phi$ bin III (with most muons exiting the detector from the side) has the smallest number of reconstructed events and the worst resolution. Bin II has the best resolution, while bins I and IV have a similar response. This is in contrast to the response in the central region [3] where the reconstruction efficiencies were roughly equal in all $\phi$ bins.
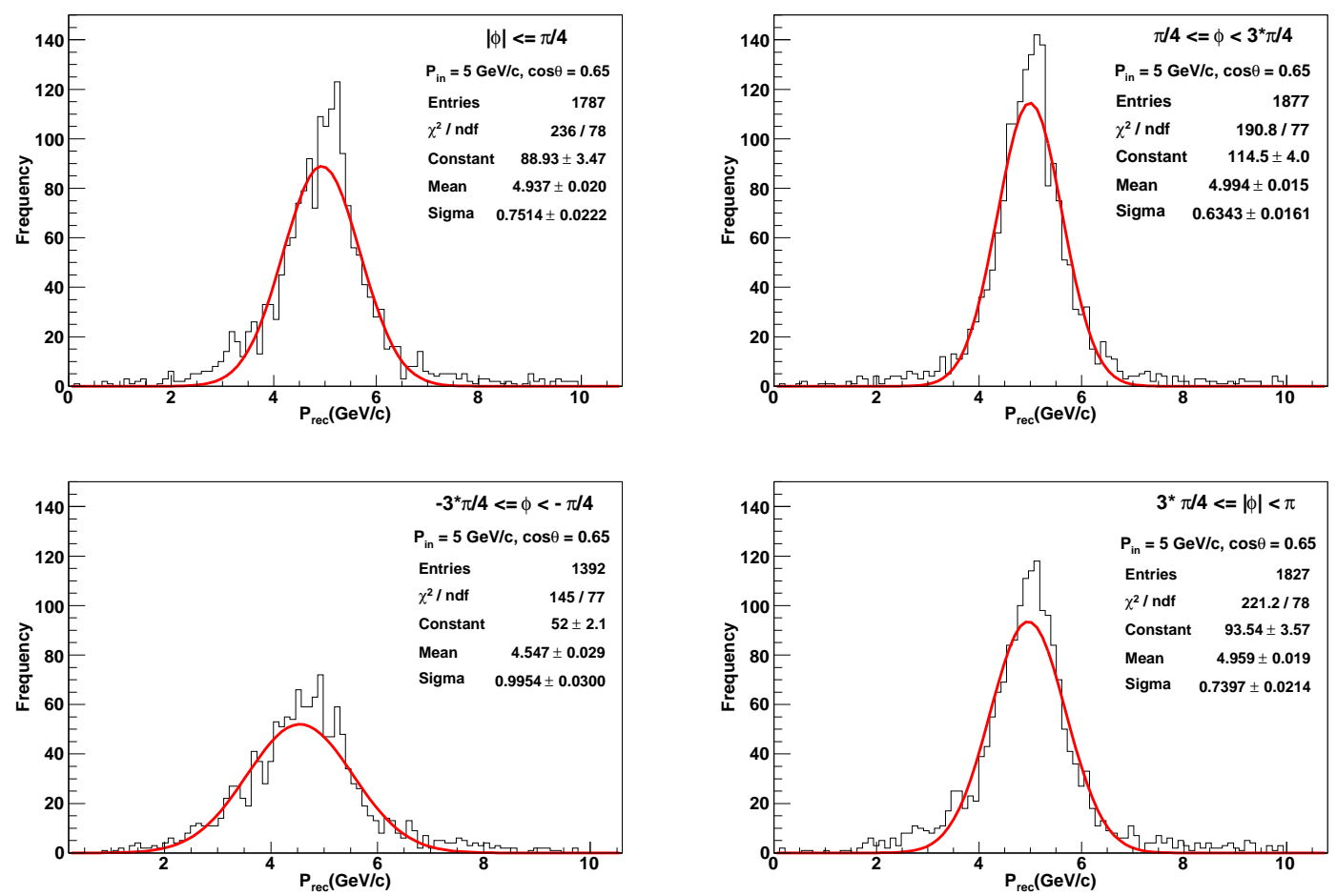

Fig. 13: Gaussian fits to reconstructed momentum distributions $P_{\text {rec }}(\mathrm{GeV} / \mathrm{c})$ for muons with fixed energy $\left(P_{i n}, \cos \theta\right)=(5 \mathrm{GeV} / \mathrm{c}, 0.65)$ in four different bins of azimuthal angle in the peripheral region. See text for details on the bins and the selection criteria used.

Similar histograms are shown in Fig. 14 for side region 9. As discussed earlier, $\phi$ bin IV has both the worst reconstruction and the worst resolution, while bin I has the best ones. Unlike the peripheral case where the bins I and IV had similar response, here bins II and III are not similar because the side region is not symmetric between these two bins: muons in bin III are more prone to exit the detector and hence the detector response is worse in both efficiency and quality of reconstruction.

The results in region 10 are similar to region 9 with interchange of bins I and IV, and bins II and III as can be easily understood from Fig. 2, However, overall the quality of reconstruction is better in region 10 by about $15 \%$ due to the nature of the forces in this region as discussed 
earlier; see Fig. 2. We shall show results for side region 9 everywhere, as being the more conservative result and simply remark on similarities/differences to be expected in region 10 .
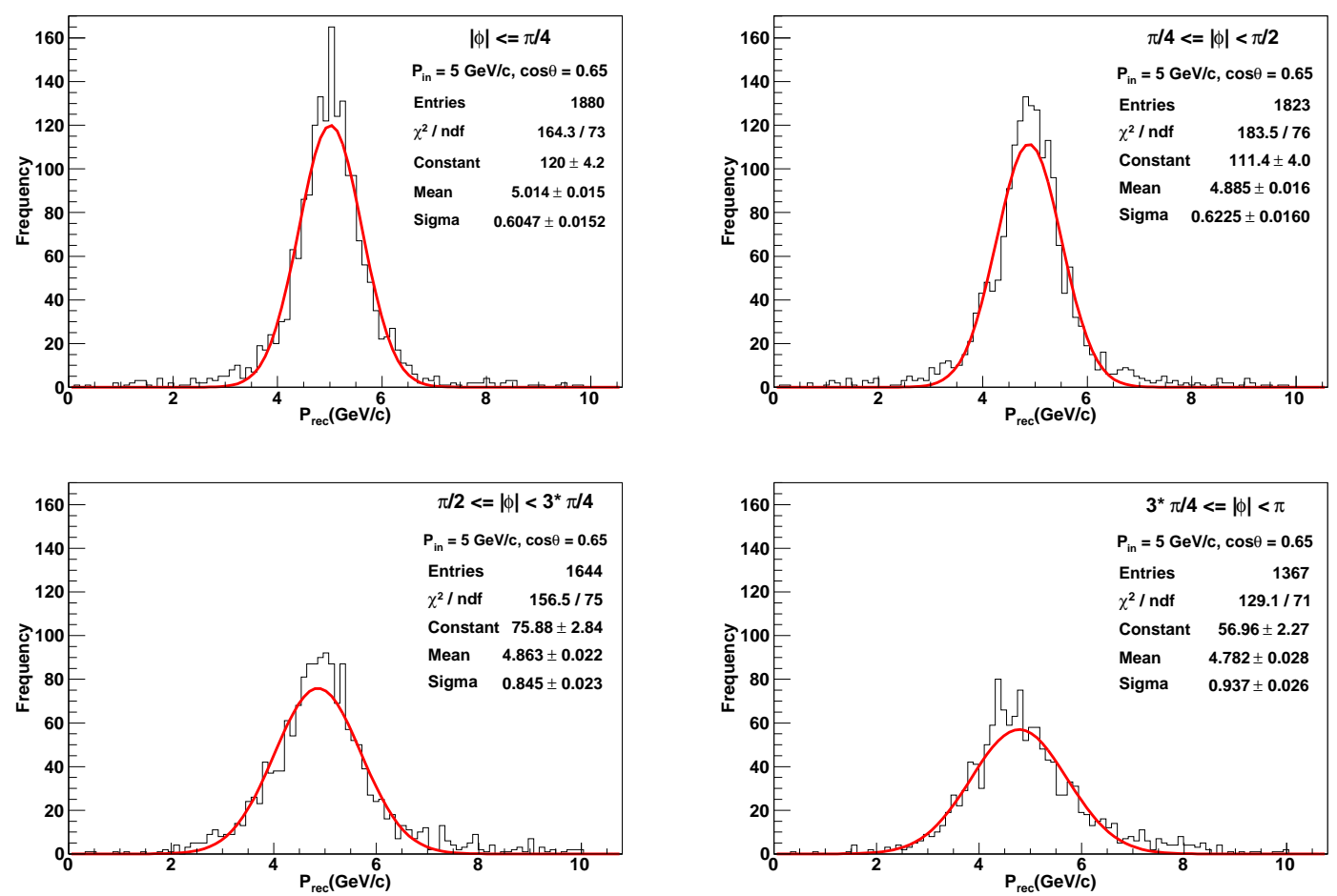

Fig. 14: Gaussian fits to reconstructed momentum distributions $P_{\text {rec }}(\mathrm{GeV} / \mathrm{c})$ for muons with fixed energy $\left(P_{i n}, \cos \theta\right)=(5 \mathrm{GeV} / \mathrm{c}, 0.65)$ in four different bins of azimuthal angle in the side region 9 . See text for details on the bins and the selection criteria used.

Fig. 15 shows the momentum resolution as a function of $P_{i n}$ in the peripheral region for the four $\phi$ bins using the selection criteria $N_{\text {hits }} / \cos \theta>n_{0}$ with $n_{0}=15,20$, for $\cos \theta=0.65$.

\subsection{Momentum Resolution as a Function of $(\theta, \phi)$}

Gaussian fits to the reconstructed momentum distribution in these regions give the reconstructed mean and RMS width $\sigma$. The momentum resolution $(R)$ is defined from these fits as,

$$
\begin{aligned}
R & =\sigma / P_{\text {in }}, \\
\text { with error } \delta R & =\delta \sigma / P_{\text {in }} .
\end{aligned}
$$

Fig. 16] shows the variation of resolution as a function of $P_{\text {in }}$ from 1 to $50 \mathrm{GeV} / \mathrm{c}$ for different values of $\cos \theta$ from 0.35 to 0.85 in the different $\phi$ bins of the peripheral region. In all bins, the momentum resolution improves with the increase of energy upto about $P_{\text {in }} \sim 6 \mathrm{GeV} / \mathrm{c}$ as the number of hits increases, but worsens at higher momenta since the particle then begins to exit the detector. This effect is considerable in the $\phi$ bin III which therefore has the worst resolution while $\phi$ bin II has the best resolution, as expected from the earlier discussions. In general, the resolution improves for more vertical angles (larger $\cos \theta$ ) as the number of hits in a track increases. 

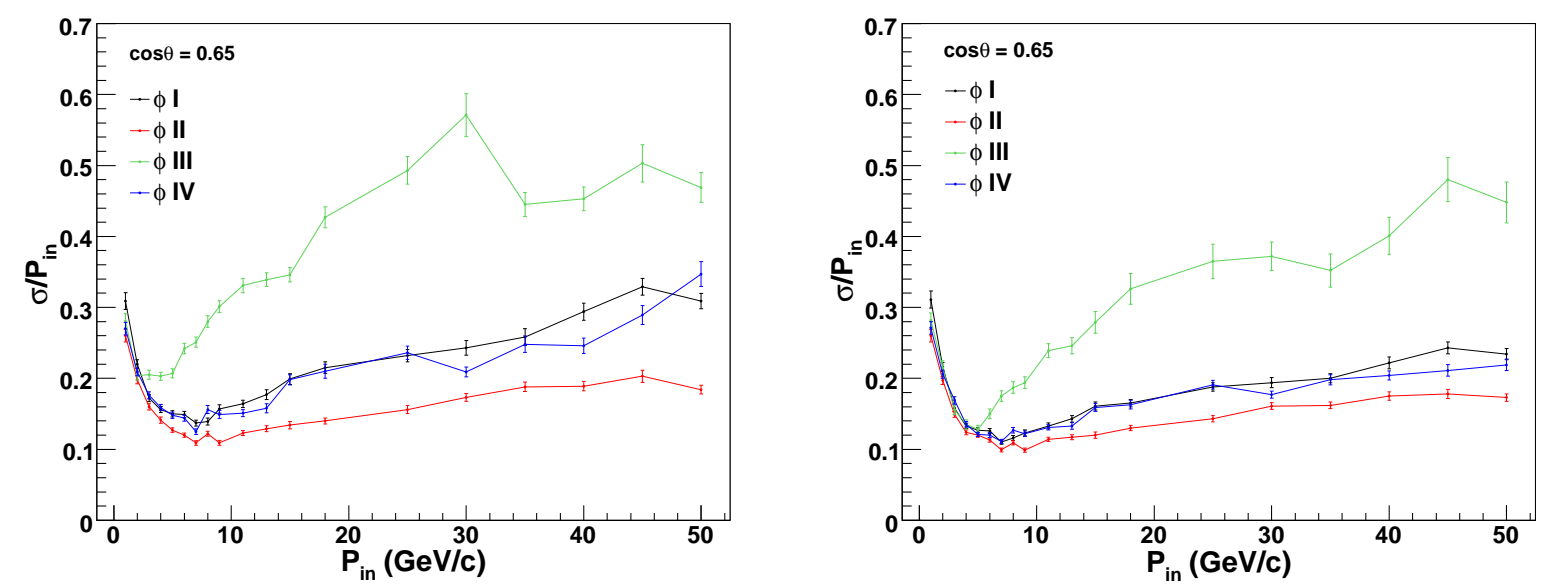

Fig. 15: Muon resolution in the peripheral region as a function of input momentum $P_{\text {in }}$ $(\mathrm{GeV} / \mathrm{c})$ for $\cos \theta=0.65$ in different bins of $\phi$ with $N_{\text {hits }} / \cos \theta>n_{0}$ cut, where $n_{0}=15(20)$ left (right).

Fig. 17 shows similar results for the side region 9. Again, it is observed that for all the angles and energies, $\phi$ bin I has the best response while the resolutions worsens in bins III and IV. Results in region 10 are similar to those in region 9 with interchange of response in $\phi$ bins (I, IV) and (II, III), but with a few percent better resolution in all cases.

The resolution for a given $P_{i n}$ is marginally better in the side region than in the peripheral region due to the somewhat larger and uniform magnetic field. A detailed comparison of the response in different regions will be presented in the next section.

\section{Comparison of Muon Response in Different Regions of ICAL}

We compare the muon response in the peripheral and side regions with that in the central region as presented in Ref. [3].

For all choices of selection criteria, the reconstruction and cid efficiencies in the central region are better than either the peripheral or side region as shown in Fig. 18 for $\cos \theta=0.65$; however, for input momenta upto $P_{i n} \sim 8 \mathrm{GeV} / \mathrm{c}$, the central and side region cid efficiencies are comparable. Note that applying more stringent selection criteria in order to improve the momentum resolution in the peripheral and side regions (and hence overall resolution of the detector) will further worsen the reconstruction efficiencies in these regions.

In addition, the angular resolutions are very similar between the peripheral and side regions, as can be seen from Fig. 12 and are in fact similar to those obtained earlier in the central region [3].

The comparison of the $\phi$-averaged peripheral and side region momentum resolutions as a function of input momentum $P_{\text {in }}$ from 1 to $15 \mathrm{GeV} / \mathrm{c}$ is shown in Fig. 19 for $\cos \theta=$ $0.45,0.65,0.85$. We have also shown the $\phi$-averaged central region results [3] in the same plots. The criterion of a single reconstructed track only was also applied to the central region, but no constraint was placed on $N_{\text {hits }}$. While the side region resolutions are only marginally better than those in the peripheral region, the central region gives the best resolution, as 

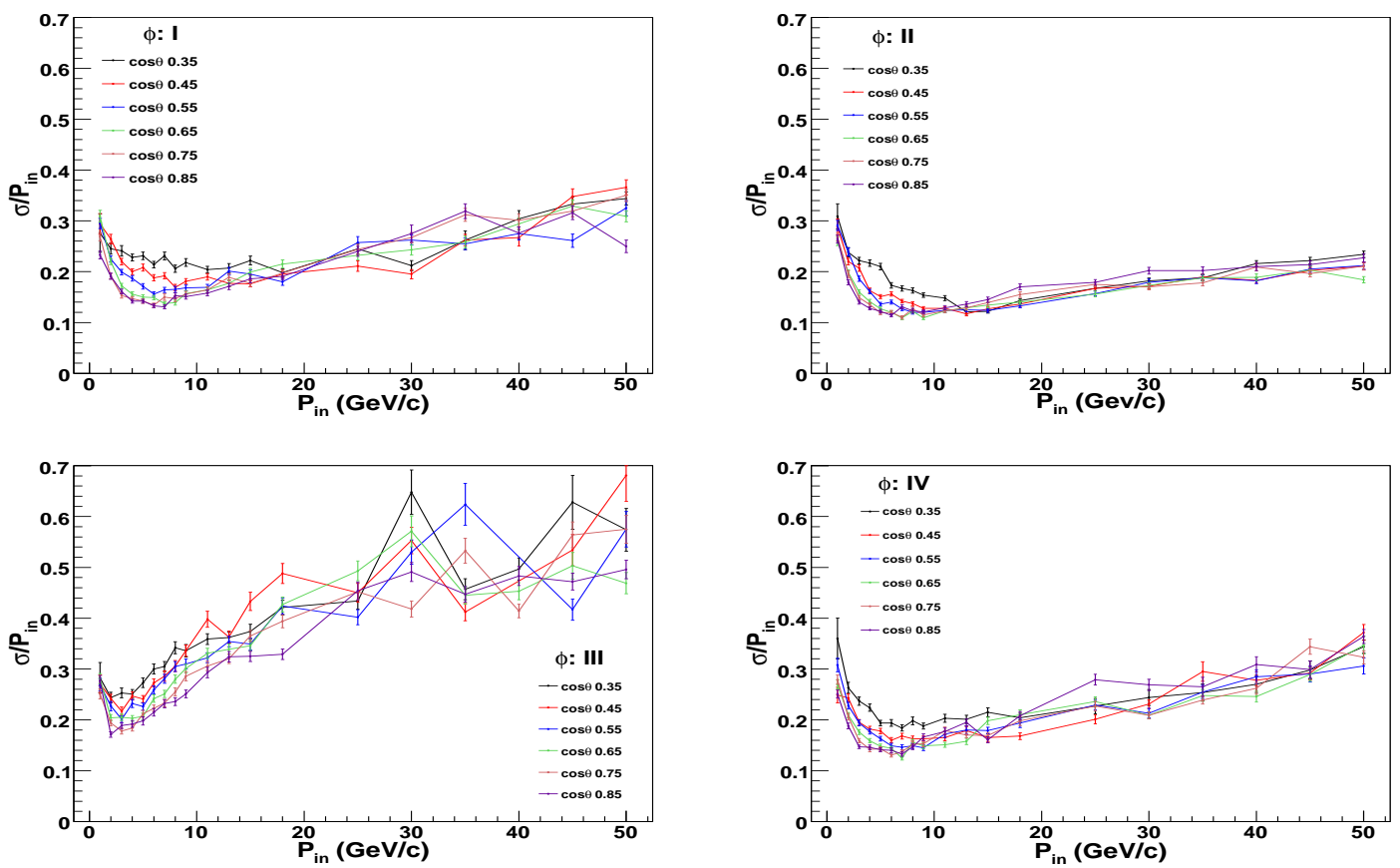

Fig. 16: Muon resolution in the peripheral region as a function of input momentum $P_{\text {in }}$ $(\mathrm{GeV} / \mathrm{c})$ for different values of $\cos \theta$ in different bins of $\phi$.
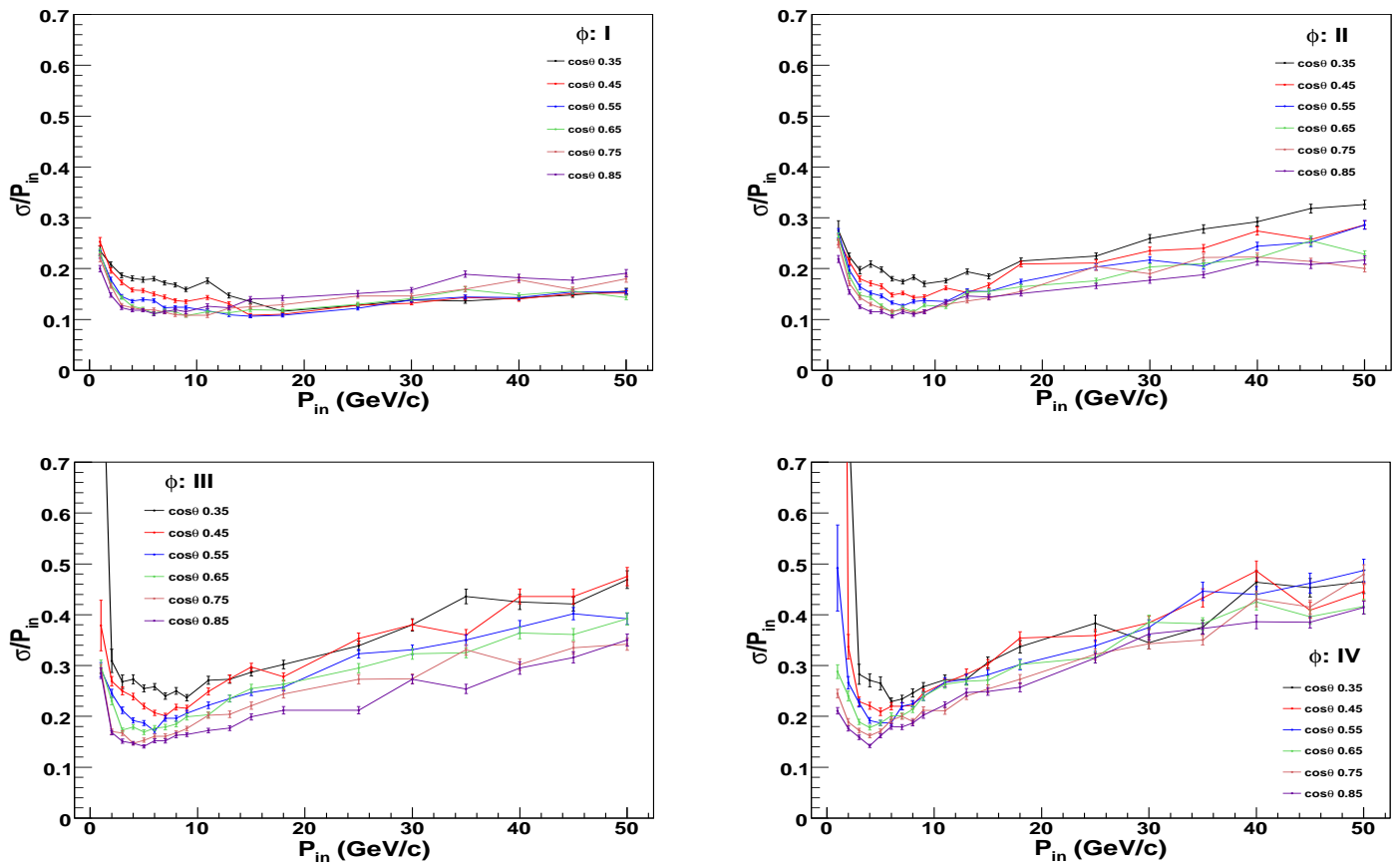

Fig. 17: Muon resolution in the side region 9 as a function of input momentum $P_{\text {in }}(\mathrm{GeV} / \mathrm{c})$ for different values of $\cos \theta$ in different bins of $\phi$. 

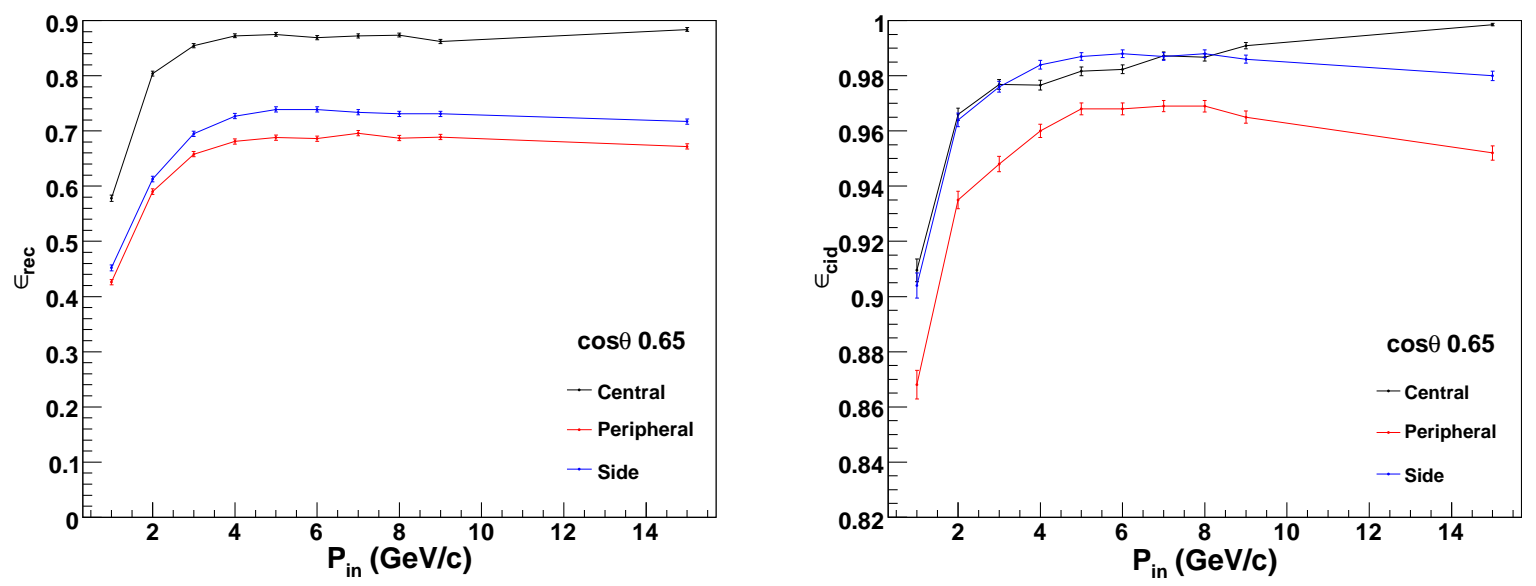

Fig. 18: Comparison of reconstruction (left) and cid efficiency (right) of central, peripheral and side regions as a function of $P_{\text {in }}(\mathrm{GeV} / \mathrm{c})$ at $\cos \theta=0.65$. Note that the y-axis scales are different for the two plots.

expected. However, we note that the results are $\phi$ averaged and so the resolutions can be much improved in the peripheral and side regions depending on the $\phi$ bin chosen. The peripheral and side region resolutions can be improved by changing the selection criteria at the cost of reconstruction efficiency. The resolutions in all regions are comparable at low momenta, $P_{\text {in }} \leq 3 \mathrm{GeV} / \mathrm{c}$, since almost all tracks are fully contained in this case.
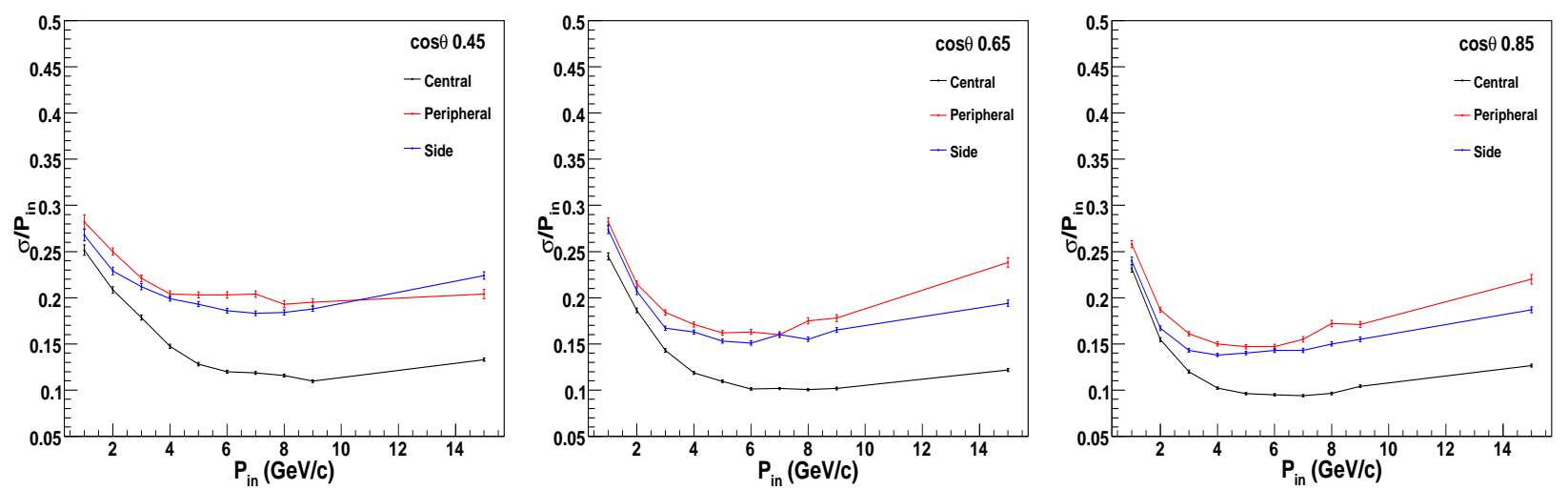

Fig. 19: Comparison of resolutions in peripheral and side region 9 as a function of the input momentum $P_{\text {in }}(\mathrm{GeV} / \mathrm{c})$ along with earlier results in the central region [3] for different values of $\cos \theta=0.45,0.65,0.85$.

\section{Discussions and Conclusion}

The goal of the proposed ICAL detector is to study neutrino oscillations using atmospheric neutrinos. It is more sensitive to muons and hence the physics will focus on charged current scattering of $\nu_{\mu}\left(\bar{\nu}_{\mu}\right)$ in the detector. Hence a simulations study of the response of ICAL 
to muons is crucial. The ICAL geometry was simulated using GEANT4 software and the detector response was studied for muons with momenta from 1 to $50 \mathrm{GeV} / \mathrm{c}$, polar angle $\cos \theta \geq 0.35$ and smeared over all azimuthal angles, $-\pi \leq \phi \leq \pi$. In the current study, muons were generated in the peripheral and side region of the ICAL detector where the magnetic field is non-uniform in both magnitude and direction and where edge effects are important. The study showed that a crucial selection criterion on the number of hits $N_{\text {hits }} / \cos \theta>n_{0}$ for partially contained tracks was necessary to achieve good detection efficiency. The magnetic field and the detector geometry break the azimuthal symmetry; hence the muon response was analysed in different $\phi$ bins.

Results using $N_{\text {hits }} / \cos \theta>15$ show that the best momentum resolutions of about $10-15 \%$ are obtained in bin II $(\pi / 4 \leq \phi<3 \pi / 4)$ at input momenta of $P_{\text {in }} \geq 4 \mathrm{GeV} / \mathrm{c}$ in the peripheral region and in bins I and II $(|\phi| \leq \pi / 4$ and $\pi / 4<|\phi| \leq \pi / 2)$ in Side region 9 (see Figs. 2 and 3 for definitions of these regions).

Also, $\phi$-averaged results are obtained with $N_{\text {hits }} / \cos \theta>15$ for the reconstruction efficiency, charge identification efficiency and momentum resolution as shown in Figs. 18 and 19 for the peripheral and side regions of the ICAL detector in comparison with earlier results in the central region [3].

A reconstruction efficiency of about $60-70 \%$ and a correct charge identification of about $97 \%$ of the reconstructed muons was obtained for $P_{\text {in }} \geq 4 \mathrm{GeV} / \mathrm{c}$ and this decreased to about $90 \%$ for higher momenta $P_{\text {in }} \sim 50 \mathrm{GeV} / \mathrm{c}$ in both regions. Average (over $\phi$ ) resolutions obtained are between $15-25 \%$ over $P_{\text {in }}=1-15 \mathrm{GeV} / \mathrm{c}$ in the peripheral region and marginally better in the side region, with the central region response being the best. Note that these responses are relevant for studies such as precision measurement of neutrino oscillation parameters or the mass hierarchy determination with ICAL. For the case of physics studies such as rock muons or cosmic ray muons, the response in only certain $\phi$ bins are relevant since the muons in these cases are always entering the detector from outside; for this reason, the performance will be better than the averages shown here.

In contrast, good angular resolution of better than a degree for $P_{\text {in }} \geq 4 \mathrm{GeV} / \mathrm{c}$ is obtained in the peripheral and side regions, which is comparable to that in the central region.

The simulations indicate that the detector has a good response to muons, with reconstruction of momentum with $15-24 \%$ resolution, direction reconstruction of about a degree for muon energies greater than $4 \mathrm{GeV}$ and charge identification of about $97 \%$. While fully contained events are reconstructed with the same efficiency as in the central region, only those partially contained ones which have at least $N_{\text {hits }} / \cos \theta>n_{0}$ in their tracks, $n_{0} \sim 15$, are well reconstructed in the simulations. This implies a loss of reconstruction efficiency due to this criterion. However, the number of events reconstructed in these regions, which is expected to be about $50 \%$ from naive considerations of detector geometry, is about $60-70 \%$, due to the effect of the magnetic field, which increases the recontruction efficiency in the peripheral region.

Acknowledgements : We thank Naba K Mondal for suggestions and support during this work. We also thank the INO simulations group for their comments and suggestions on the results; Gobinda Majumder and Asmita Redij for code-related discussions; and Shiba Behera for discussions on the magnetic field map. R. Kanishka acknowledges UGC/DST (Govt. of India) for financial support. 


\section{References}

[1] M. S. Athar et al., 2006 India-based Neutrino Observatory: Project Report Volume I, http://www.ino.tifr.res.in/ino/OpenReports/INOReport.pdf.

[2] M. M. Devi et al., Hadron energy response of the Iron Calorimeter detector at the Indiabased Neutrino Observatory, JINST 8 P11003 (2013) arXiv:1304.5115].

[3] A. Chatterjee et al., A Simulations Study of the Muon Response of the Iron Calorimeter detector at the India-based Neutrino Observatory, JINST 9 P07001 (2014) arXiv:1405.7243.

[4] T. Thakore, A. Ghosh, S. Choubey and A. Dighe, The Reach of INO for Atmospheric Neutrino Oscillation Parameters, JHEP 1305, 058 (2013) [arXiv:1303.2534[hep-ph]].

[5] A. Ghosh, T. Thakore and S. Choubey, Determining the Neutrino Mass Hierarchy with INO, T2K, NOvA and Reactor Experiments, JHEP 1304, 009 (2013) arXiv:1212.1305[hep-ph]].

[6] GEANT4 collaboration, S. Agostinelli et al. Geant4: a simulation toolkit, Nucl. Instrum. Meth. A 506, 250-303 (2003) http://geant4.cern.ch.

[7] Infolytica Corp., Electromagnetic field simulation software, http://www.infolytica.com/en/products/magnet/.

[8] R. E. Kalman, A new approach to linear filtering and prediction problems. Journal of Basic Engineering 82 (1) 35-45, (1960).

[9] B. Satyanarayana, Design and Characterisation Studies of Resistive Plate Chambers, PhD thesis, Department of Physics, IIT Bombay, PHY-PHD-10-701, (2009).

[10] Kolahal Bhattacharya, et al., INO Collaboration, Error propagation of the track model and track fitting strategy for the iron calorimeter detector in India-based neutrino observatory, Computer Physics Communications (Elsevier) 185 (12) 3259-3268, (2014). 